\title{
Luminescent properties of $\mathrm{LuPO}_{4}-\mathrm{Pr}$ and $\mathrm{LuPO}_{4}-\mathrm{Eu}$ nanoparticles
}

\author{
V. Vistovskyy ${ }^{1,1}$, T. Malyi ${ }^{1}$, A. Vas'kiv ${ }^{1}$, M. Chylii ${ }^{1}$,
}

N. Mitina ${ }^{2}$, A. Zaichenko², A. Gektin ${ }^{3}$, A. Voloshinovskii ${ }^{1}$

${ }^{1}$ Ivan Franko National University of Lviv, 8 Kyryla i Mefodiya, 79005, Lviv, Ukraine

${ }^{2}$ Lviv Polytechnic National University, 12 S. Bandera, 79013, Lviv, Ukraine

${ }^{3}$ Institute for Scintillation Materials, NAS of Ukraine, 60 Lenina Ave, 61001

Kharkiv, Ukraine

\begin{abstract}
Spectral-luminescence parameters of $\mathrm{LuPO}_{4}$-Eu and $\mathrm{LuPO}_{4}$-Pr nanoparticles of different sizes are studied upon excitation by the synchrotron radiation with photon energies 4-40 eV. Influence of the nanoparticle size on $\mathrm{Eu}^{3+}$ and $\mathrm{Pr}^{3+}$ impurity luminescence is analyzed for intracenter and recombination excitation. It is shown that the luminescence intensity of impurities in the case of recombination excitation significantly stronger decreases with decreasing of nanoparticle size compared to intracenter excitation. This feature is explained by the influence of thermalization length to nanoparticle size ratio on the recombination luminescence. Electron recombination luminescence inherent for $\mathrm{LuPO}_{4}$-Eu nanoparticles shows a weaker dependence on the nanoparticle size than the hole one in $\mathrm{LuPO}_{4}-\mathrm{Pr}$ nanoparticles. The difference between energy states of praseodymium impurity ions in nanoparticles of different sizes is revealed.
\end{abstract}

Keywords: $\mathrm{LuPO}_{4}$-Eu nanoparticles, $\mathrm{LuPO}_{4}$-Pr nanoparticles, recombination luminescence

\section{Introduction}

The attention to luminescence of nanoparticles upon high energy excitation is caused by the prospect of their use as nanoscintillators for radiotherapy and photodynamic radiotherapy [1] and also to create bulk nanocomposite scintillators that combine the advantages of inorganic and organic luminescent materials [2]. High light yield and fast response are important for nanocomposite scintillators based on polymeric matrix with embedded inorganic nanoparticles. In the case of

\footnotetext{
${ }^{1}$ Corresponding author e-mail: vistvv@gmail.com
} 
nanoscintillators for radiotherapy the main important parameters are spectral emission range and high light yield. Thus, for radiotherapy the emission spectrum of nanoparticles should be in the UV spectral region $(<280 \mathrm{~nm})$, where the absorption bands of proteins are located, that will allow their destruction. For photodynamic therapy the emission spectrum of nanoparticles has to be well matched to the absorption spectrum of photosensitizers. Photosensitizers produce singlet oxygen that attacks key structural entities within the targeted cells.

Considering these requirements for nanoscintillators, Lu-containing oxide compounds doped with luminescent $\mathrm{Pr}^{3+}$ and $\mathrm{Eu}^{3+}$ impurities are of interest. Praseodymium ions offer $5 d-4 f$-radiative transitions in the UV range $(220-270 \mathrm{~nm})$ with short decay constant $\sim 10$ ns. Europium ions emission in the red spectral range 580-715 nm is consistent with absorption spectra of the photosensitizer hematoporphyrin. The fact that lutetium-containing materials possess a high capacity for absorption of radiation and usually show high light yield causes the choice of studied compound.

For successful creation of nanoscintillators it is necessary to reveal the dependence of luminescence intensity of nanoparticles on their size and the energy of excitation photons. The decrease of nanoparticle sizes causes the increase of surface/volume ratio and consequently leads to quenching of luminescence due to nonradiative resonance energy transfer from excited states of luminescence centers to surface defects. This effect is best to observe analyzing the change of luminescence decay kinetic with decrease of nanoparticle size in the case of intracenter excitation. The decrease of nanoparticle sizes, which correlates with the increase of surface influence, leads to luminescence decay time shortening. This was well illustrated for $\mathrm{LaPO}_{4}$-Ce nanoparticles, where the noticeable shortening of cerium luminescence decay kinetics was observed at transition from microcrystals of nanocrystals [3]. This effect is observed upon the intracenter excitation. When luminescence is excited by the quanta with energy higher than forbidden band gap $\left(E_{\mathrm{g}}\right)$ in addition to the resonance energy transfer from the luminescent centers to surface defects the other size phenomena connected with charge carriers migration are expected. This is because the migration stage of electrons and holes precedes the act of their recombination on luminescence centers. Different scattering mechanisms of highenergy electrons and holes can occur depending on the exciting photons energy in the case of recombination excitation. Path length of charge carriers is determined by the scattering mechanisms. The photoelectrons created by photons with energies of $E_{\mathrm{g}}<h v<2 E_{\mathrm{g}}$ will possess the kinetic energy $K<E_{\mathrm{g}}$ that is not enough for electronelectron scattering. Such electrons are thermalized due to electron-phonon scattering. The characteristic parameter of this process is thermalization length which is of several dozen nanometers [4]. The mean free path of photoelectrons created with energy quanta $h v>2 E_{\mathrm{g}}$ due to inelastic scattering on valence electrons is about few nanometers. In the case of photoelectrons, generated by X-ray radiation, the mean free path varies from tens to hundreds of nanometers [5]. As a result for recombitation luminescence of nanoparticles it is needed to take into account the ratio between the electron path length and the nanoparticle size. If the mean free path of photoelectrons or thermalization length is comparable or larger than nanoparticle size then the luminescence intensity will decrease. These considerations were taken into 
account when the dependence of luminescence intensity on the size of nanoparticles upon band-band excitation was analyzed for $\mathrm{Lu}_{2} \mathrm{O}_{3}, \mathrm{LaPO}_{4}, \mathrm{LaF}_{3}, \mathrm{CaF}_{2}$ nanoparticles [3,6-9]. Mentioned circumstances are considered in detail for $\mathrm{LaPO}_{4}-\mathrm{Eu}$ and $\mathrm{LaPO}_{4^{-}}$ Pr nanoparticles [10]. However, $\mathrm{LaPO}_{4}$ nanoparticles with sizes less than $a<20 \mathrm{~nm}$ possess hexagonal crystalline symmetry, whereas particles with $a>20 \mathrm{~nm}$ possess monoclinic symmetry [11]. Such peculiarity can have an effect on the dependence of luminescence intensity on the size of the nanoparticles. In the case of $\mathrm{LuPO}_{4}$ nanoparticles possessing the tetragonal crystalline symmetry irrespective of their size, the effects connected with influence of charge carrier migration on luminescent parameters dependently on the nanoparticle size should be observed more clearly comparing to $\mathrm{LaPO}_{4}$ system.

\section{Experiment}

$\mathrm{LuPO}_{4}: \mathrm{Ln}\left(\mathrm{Ln}=\mathrm{Pr}^{3+}, \mathrm{Eu}^{3+}\right)$ nanoparticles were synthesized via sedimentation nucleation method from water solutions of corresponding salts. For this purpose the $9 \mathrm{ml}$ of $\mathrm{LuCl}_{3} \times 7 \mathrm{H}_{2} \mathrm{O}$ and $\mathrm{LnCl}_{3} \times 6 \mathrm{H}_{2} \mathrm{O}$ water solution was prepared. Water solution of $\mathrm{LuCl}_{3}$ and $\mathrm{LnCl}_{3}$ was slowly added with $1 \mathrm{drop} / \mathrm{s}$ rate to the solution of $\mathrm{NaH}_{2} \mathrm{PO}_{4}$ at continuous mixing. As a result a white fine-dispersed precipitate was obtained. Precipitate was separated by the centrifuge and washed out by the distilled water several times to neutral $\mathrm{pH}$ value and finally washed out by acetone. The obtained $\mathrm{LuPO}_{4}: \mathrm{Ln}$ nanoparticles were dried under vacuum to constant weight. In order to increase the nanoparticles size the synthesized particles were annealed during 2 hours at the different temperatures $\left(400,800,1200^{\circ} \mathrm{C}\right)$.

The crystalline symmetry of lattice and the size of nanoparticles were estimated via X-ray diffraction method. For X-ray diffraction studies the uniform layer of nanopowders was deposited on a substrate using X-ray-amorphous glue. The diffraction patterns (Fig. 1) were obtained by the means of STOE STADI P diffractometer. Accordingly to X-ray diffraction data the unannealed $\mathrm{LuPO}_{4}$ - $\mathrm{Ln}$ nanopowders are amorphous-fractal. The crystalline structure of $\mathrm{LuPO}_{4}$ phase is tetragonal of $14_{1} /$ amd space group. The mean size of particles is less than $5 \mathrm{~nm}$. Nanoparticles annealed at $400^{\circ} \mathrm{C}, 800^{\circ} \mathrm{C}$ and $1200^{\circ} \mathrm{C}$ are monophasic with the mean size of 8,12 and 35 nanometers, respectively. The parameters of crystalline lattices and the mean sizes of nanoparticles are shown in Table 1.

Table 1. Size and symmetry of $\mathrm{LuPO}_{4}$-Ln nanoparticles according to X-ray diffraction measurements.

\begin{tabular}{lcc}
\hline $\begin{array}{l}\text { Samples of } \\
\mathrm{LuPO}_{4} \text { - } \mathrm{Ln}\end{array}$ & Symmetry of lattice & $\begin{array}{c}\text { Mean grain size, } \\
a(\mathrm{~nm}) ;\end{array}$ \\
\hline \hline Unannealed & $\begin{array}{l}\text { amorphous-fractal, } \\
\text { tetragonal } I 4_{1} / \text { amd }\end{array}$ & $<5$ \\
\hline Annealed & tetragonal & $8 \pm 5$ \\
\hline
\end{tabular}




\begin{tabular}{lcc}
\hline at $400^{\circ} \mathrm{C}$ & I4 $/$ /amd & \\
\hline Annealed & tetragonal & $12 \pm 5$ \\
at $800^{\circ} \mathrm{C}$ & $I 4_{1} /$ amd & \\
\hline Annealed at $1200^{\circ} \mathrm{C}$ & $\begin{array}{c}\text { tetragonal } \\
\text { I }\end{array}$ /amd & $35 \pm 12$ \\
\hline
\end{tabular}

Spectral-kinetic measurements upon excitation with synchrotron radiation quanta were performed at SUPERLUMI station of HASYLAB in Hamburg (Germany). Synchrotron radiation from accelerator of positrons DORIS III was used as excitation light. Luminescence spectra were measured using secondary monochromatorspectrograph ARC "Spectra Pro 308” equipped with a CCD the detector. Excitation light wavelength was specified by means of primary $2 \mathrm{~m}$ monochromator with spectral resolution ability of $3.2 \AA$ [12].

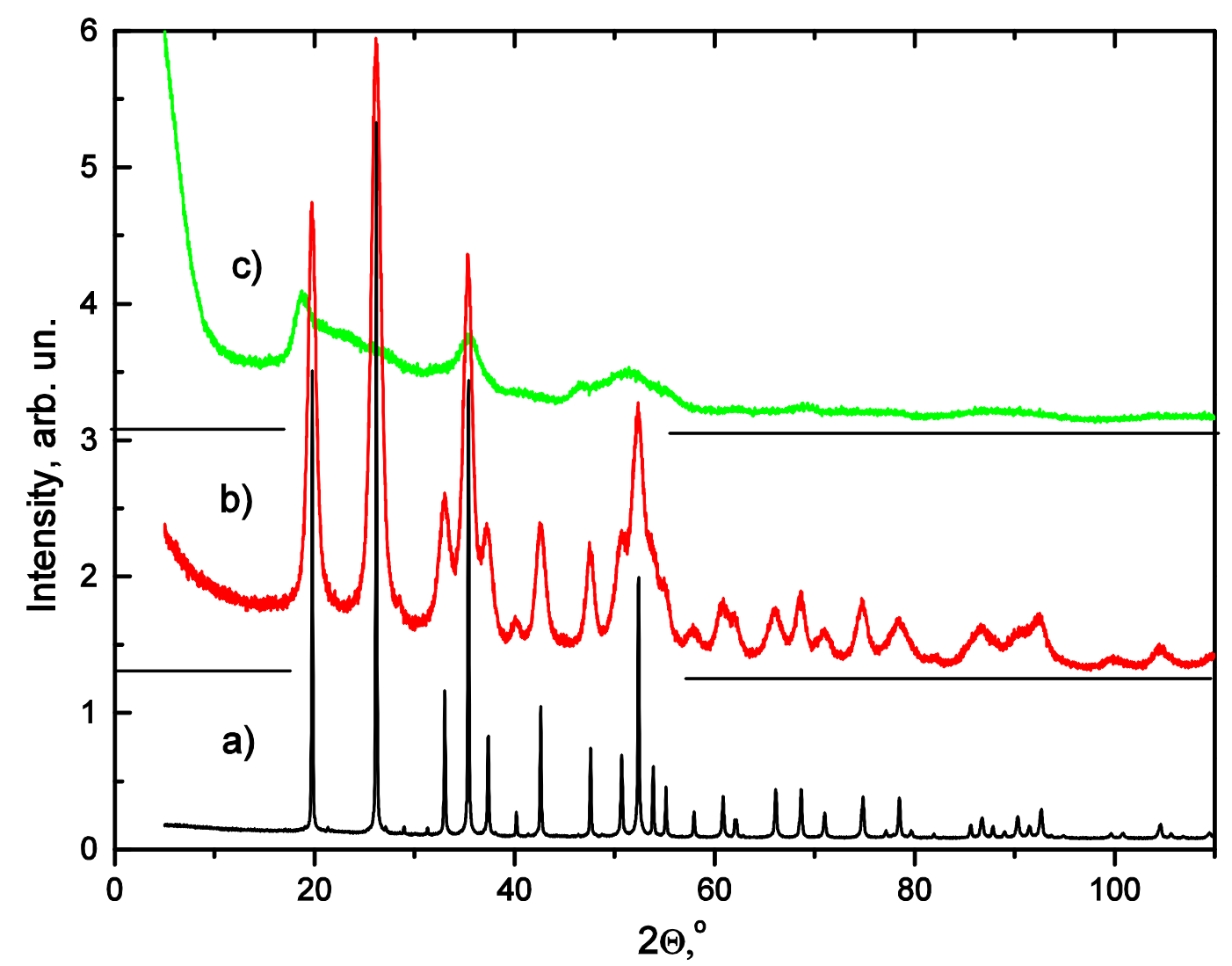

Fig. 1. Diffraction patterns of $\mathrm{LuPO}_{4}$-Pr nanoparticles annealed at $1200{ }^{\circ} \mathrm{C}$ (a), $800{ }^{\circ} \mathrm{C}$ (b) and unannealed samples (c).

\section{Result and discussion}

\subsection{Luminescent properties of $\mathrm{LuPO}_{4}$-Eu nanoparticles}

Luminescence spectra of $\mathrm{LuPO}_{4}$-Eu nanoparticles upon photoexcitation by quanta with energy of $5.8 \mathrm{eV}$ are shown in Fig. 2. These spectra consist of 
luminescence bands corresponding to $4 \mathrm{f}$-4f-transitions in $\mathrm{Eu}^{3+}$ ions from the lowest excited ${ }^{5} D_{0}$ state to the ${ }^{7} F_{J}(J=1,2,3,4)$ level split by the spin-orbit interaction.

The structure of luminescence bands for nanoparticles with mean size of 35 and $12 \mathrm{~nm}$ is typical for tetragonal crystalline symmetry. Broadening of europium emission bands and change of their relative intensity is observed for $5 \mathrm{~nm}$ nanoparticles. Such situation is common for small size nanoparticles and is explained by the influence of crystal field fluctuations on the impurities [13].

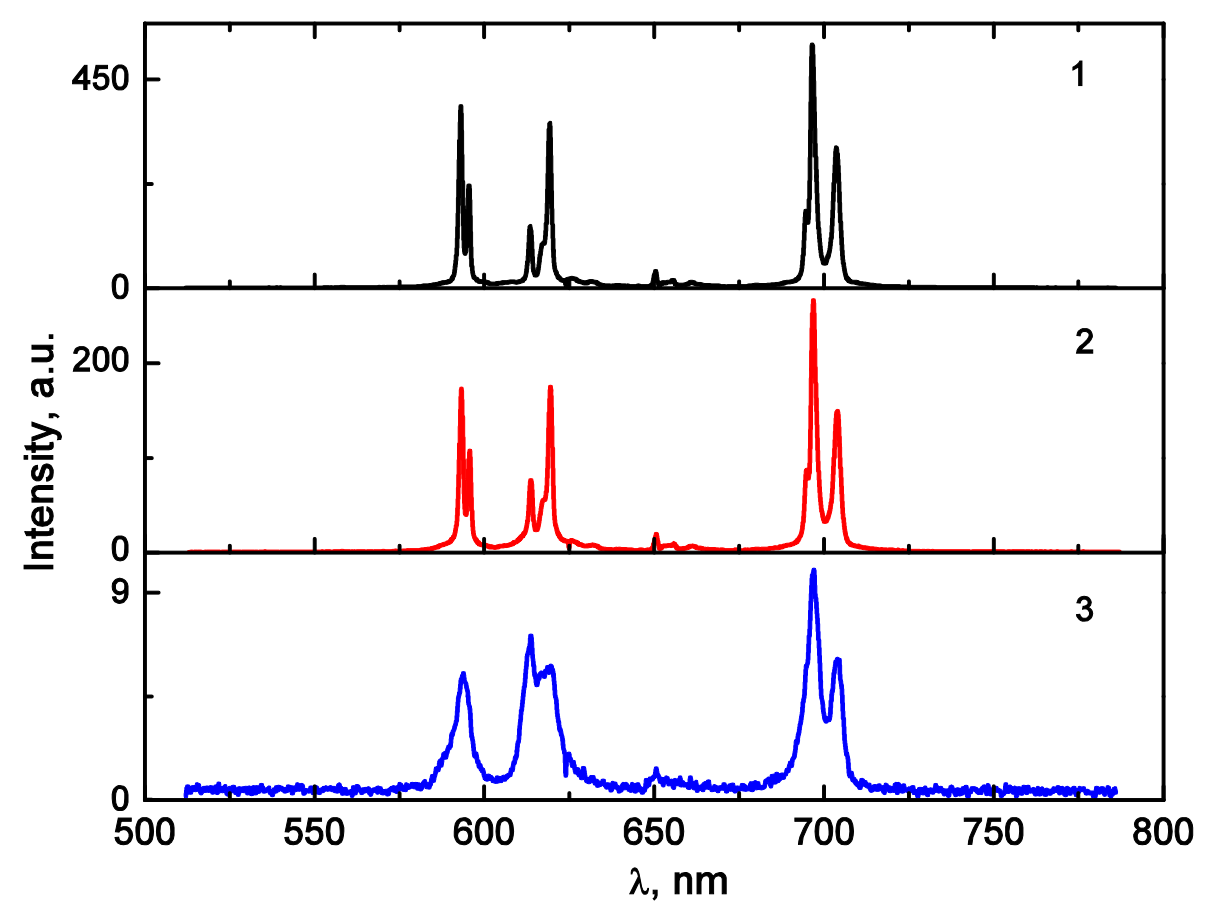

Fig. 2. Luminescence spectra of $\mathrm{LuPO}_{4}-\mathrm{Eu}$ nanoparticles with different size upon excitation in the charge transfer band $\left(\lambda_{\text {exc }}=210 \mathrm{~nm}\right)$. Curves: $1-a=35 \mathrm{~nm}$, $2-a=12 \mathrm{~nm}, 3-a<5 \mathrm{~nm}$. T $=300 \mathrm{~K}$

Luminescence excitation spectra of $\mathrm{Eu}^{3+}$ ions $\left(\lambda_{\mathrm{em}}=593 \mathrm{~nm}\right)$ in $\mathrm{LuPO}_{4}-\mathrm{Eu}$ nanoparticles of different sizes are shown in Fig. 3. Several ranges in europium luminescence excitation spectra can be identified: (i) charge transfer band from $\mathrm{O}^{2-}$ to $\mathrm{Eu}^{3+}$ with maximum at $5.8 \mathrm{eV}\left(E^{\mathrm{CT}}\right)$, (ii) the band corresponding to $4 \mathrm{f}-5 \mathrm{~d}$ transition in $\mathrm{Eu}^{3+}$ ion with a maximum at $8.1 \mathrm{eV}$, (iii) the range of optical creation of self-trapped exciton (STE) with maximum at $8.8 \mathrm{eV}\left(E_{\text {exc }}\right.$ ), (iv) the range of electron-hole pairs creation $\left(E_{\mathrm{g}}<h v<E_{t h}^{C T}\right)$ and (v) the range of electronic excitations multiplication. The electronic multiplication in $\mathrm{LuPO}_{4}$-Eu can occur with creation of secondary charge transfer excitations and secondary electron-hole pairs. Energy threshold for the first process is expected at $E_{t h}^{C T}=E_{\mathrm{g}}+E_{\text {min }}^{C T} \approx 14 \mathrm{eV}$ (where $E_{\text {min }}^{C T}-$ low energy edge of charge transfer band) and for second one at $h v>2 E_{\mathrm{g}} \approx 18 \mathrm{eV}$. 


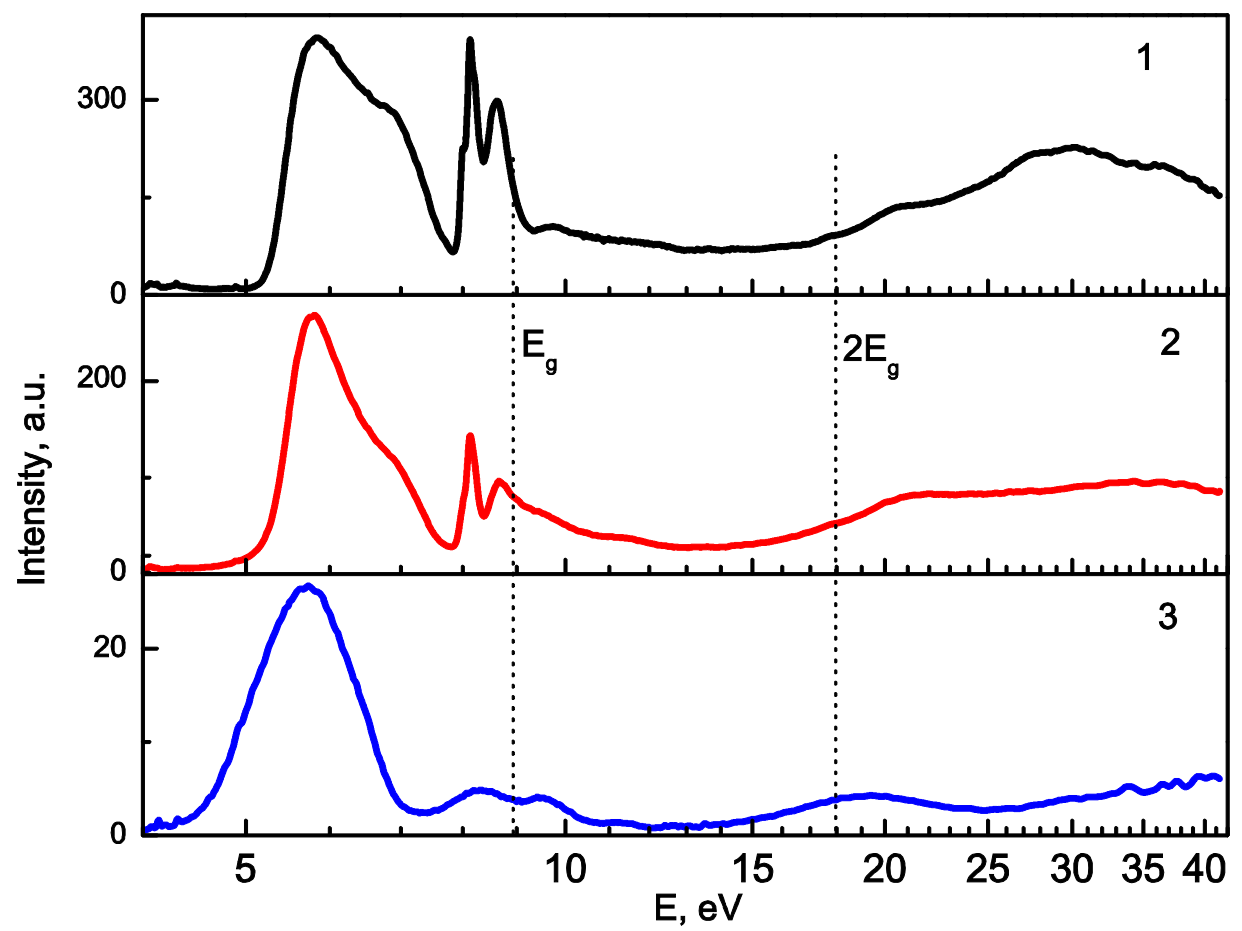

Fig. 3. Luminescence excitation spectra $\left(\lambda_{e m}=593 \mathrm{~nm}\right)$ of $\mathrm{LuPO}_{4}-\mathrm{Eu}$ nanoparticles with different size. Curves: $1-a=35 \mathrm{~nm}, 2-a=12 \mathrm{~nm}, 3-a<5 \mathrm{~nm}$. T $=300 \mathrm{~K}$

The general tendency of luminescence intensity decrease with the nanoparticle size reduce is observed. Least sensitive to the particle size reduction is the intensity of luminescence excited in the charge transfer band (Fig. 4, curve 1) and accordingly in the range of electronic multiplication with creation of secondary charge transfer excitations (curve 5). Very significant luminescence quenching is observed for excitation in the 4f-5d absorption band of $\mathrm{Eu}^{3+}$ ion (Fig. 4, curve 2). This is unexpected result for intracenter excitation [10], but it is difficult to make any conclusions from this fact, because 5d-levels are very sensitive to crystal field and at the nanoparticle size decrease the $5 \mathrm{~d}$-levels can be shifted in range of higher energy causing the probable energy overlapping of $4 \mathrm{f}-5 \mathrm{~d}$ and exciton (or band-to-band) absorption.

Luminescence intensity is also very sensitive to the nanoparticle size under excitation in exciton absorption range (Fig. 4, curve 3), but it is not so critical comparing to that for the excitation energy at $h v>E_{\mathrm{g}}$, where the strongest decrease of luminescence intensity is observed (curve 4), because the thermalization length of electrons has the major role in recombination processes upon the band-to-band excitation. The sharpest decrease of luminescence intensity upon the excitation in range of electron-hole creation was also observed in $\mathrm{Lu}_{2} \mathrm{O}_{3}$-Eu and $\mathrm{LaPO}_{4}$ - $\mathrm{Ln}[7,10]$. Decrease of luminescence intensity for excitation in range of electronic multiplication with creation of secondary electron-hole pairs is expected to be same as for the case of their direct creation, however comparing curves 4 and 6 it can be seen that luminescence excited by quanta with energy of $30 \mathrm{eV}$ reveals weaker size dependence. Possibly it is due to the processes of electronic excitation multiplication involving the creation of secondary charge transfer excitations that can give contribution in this range. 


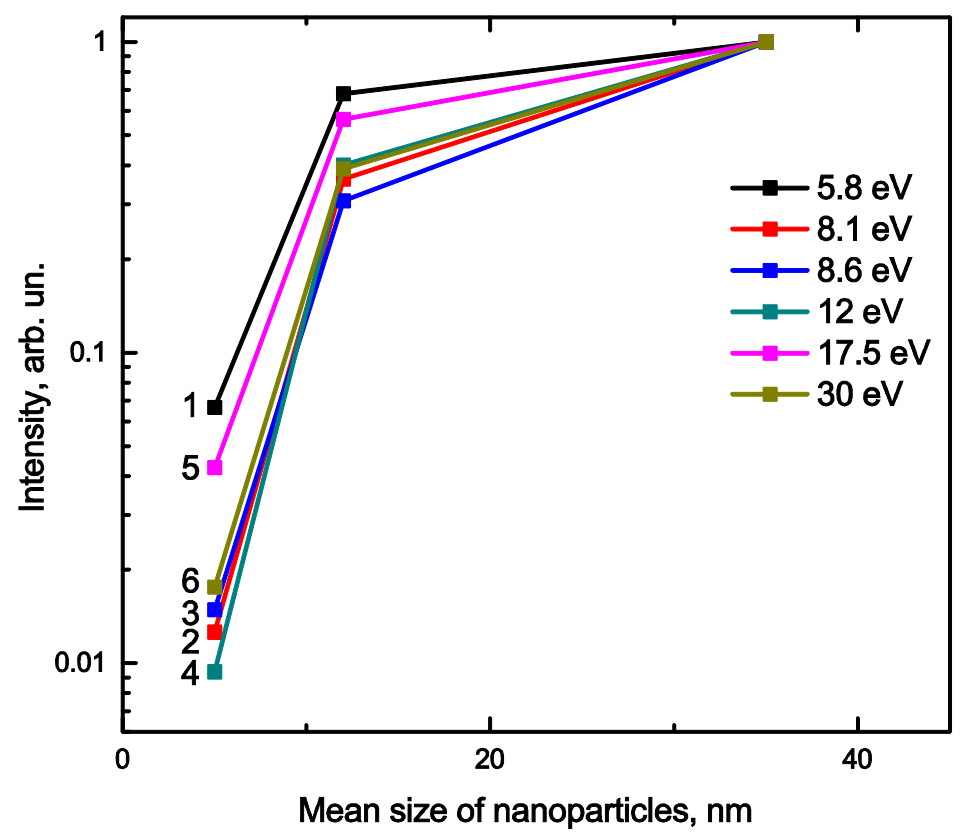

Fig. 4. Impurity luminescence intensity dependences in $\mathrm{LuPO}_{4}-\mathrm{Eu}$ nanoparticles of different size for different excitation energies. Curves: 1 - excitation in the charge transfer band $(5.8 \mathrm{eV}) ; 2$ - excitation in the $4 f-5 d$ transitions band of $\mathrm{Eu}^{3+}$ ion $(8.1 \mathrm{eV}) ; 3$ - excitation in the range of exciton absorption $(8.6 \mathrm{eV}) ; 4$ - excitation in the range of electron-hole pairs creation $(12 \mathrm{eV}) ; 5$ - excitation in the electronic excitation multiplication range involving the secondary charge transfer processes $(17.5 \mathrm{eV}) ; 6$ - excitation in the electronic excitation multiplication range involving the creation of secondary electron-hole pairs $(30 \mathrm{eV})$.

\subsection{Luminescent properties of $\mathrm{LuPO}_{4}$-Pr nanoparticles}

Luminescence spectra of $\mathrm{LuPO}_{4}$-Pr nanoparticles with different sizes upon excitation in the praseodymium ion intracenter absorption band are shown in Fig. 5. In these luminescence spectra the emission bands in the range of 225-290 nm with maxima at 236, 246, 264 and $274 \mathrm{~nm}$, corresponding to radiative transitions from $5 d$ states to ${ }^{3} \mathrm{H}_{\mathrm{J}}$ and ${ }^{3} \mathrm{~F}_{\mathrm{J}}$ ground states of praseodymium ion, are observed. The overall structure of luminescence spectra for nanoparticles with different sizes is practically identical. The sharper separation of single luminescence bands is observed with the increase of nanoparticle size. 


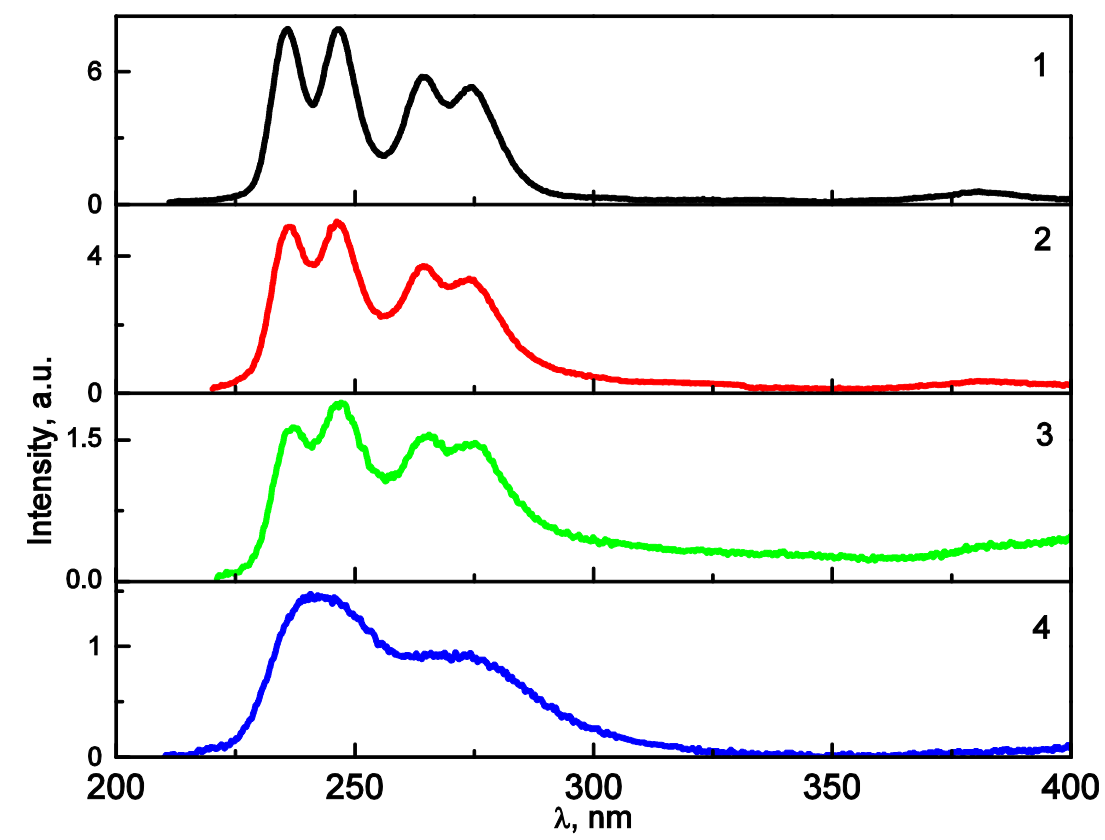

Fig. 5. Luminescence spectra of $\mathrm{LuPO}_{4}$-Pr nanoparticles with different sizes upon the excitation in the $4 f-5 d$ absorption range of $\operatorname{Pr}^{3+}$ ions $\left(\lambda_{\text {exc }}=213 \mathrm{~nm}\right)$. Curves: $1-$ $a=35 \mathrm{~nm}, 2-a=12 \mathrm{~nm}, 3-a=8 \mathrm{~nm}, 4-a<5 \mathrm{~nm}$. T $=300 \mathrm{~K}$

Luminescence excitation spectra of praseodymium ions in $\mathrm{LuPO}_{4}-\mathrm{Pr}$ nanoparticles are presented on Fig. 6 . In the low-energy spectrum range ( $h v<E_{\mathrm{g}}$ ) the excitation bands with maxima at $5.4 \mathrm{eV}, 5.85 \mathrm{eV}$ and $6.54 \mathrm{eV}$ corresponding to intracenter 4f-5d-absorption transitions in $\mathrm{Pr}^{3+}$ ions are observed. In the energy range of $E_{\mathrm{g}}<h v<2 E_{\mathrm{g}}$ the excitation of praseodymium ions occurs due to recombination mechanism via energy transfer from electron-hole pairs of $\mathrm{LuPO}_{4}$ matrix. The luminescence intensity increasing threshold in excitation spectra starting at $h v>2 E_{\mathrm{g}}$ corresponds to the electronic excitation multiplication process with creation of secondary electron-hole pairs. 


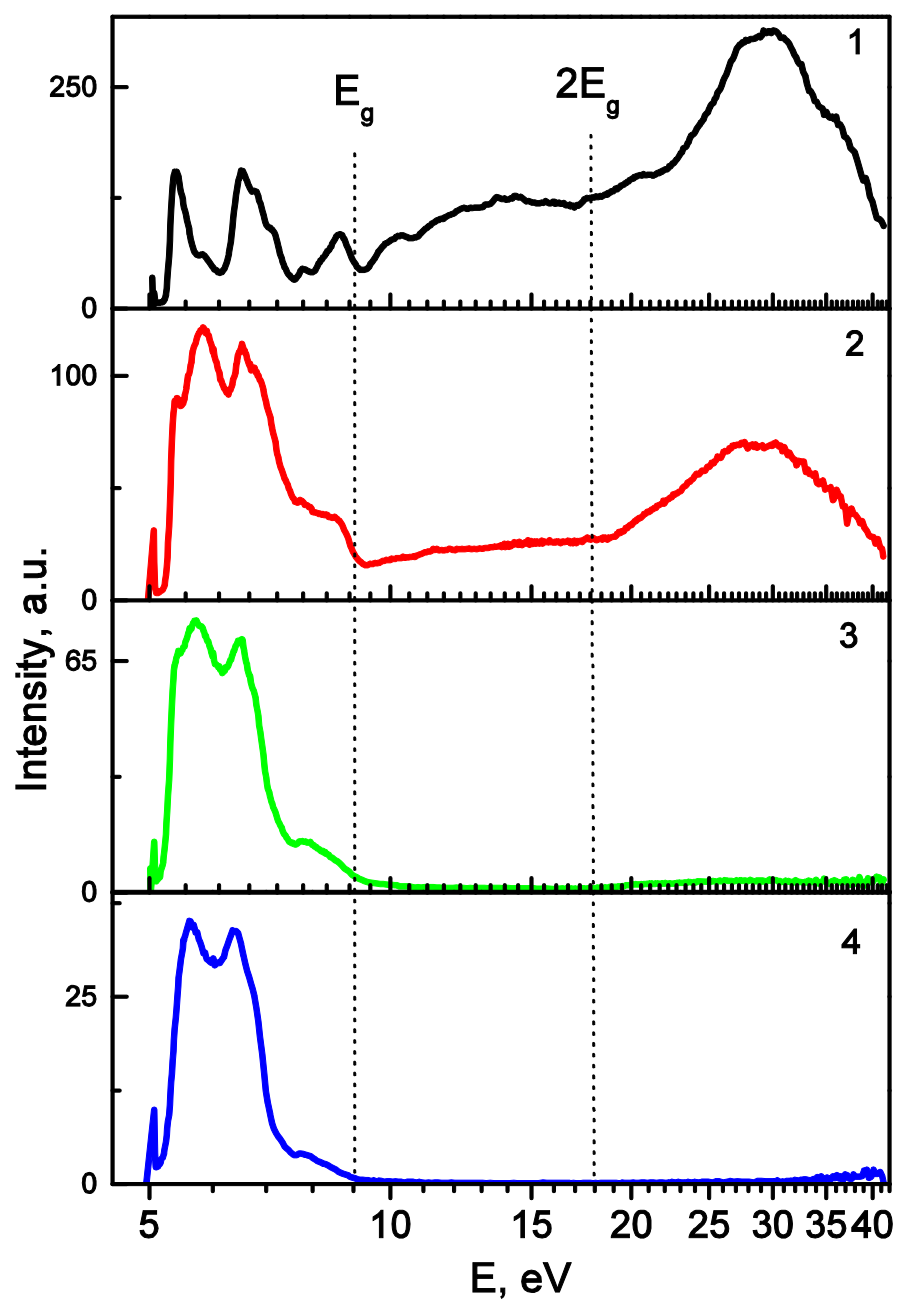

Fig. 6. Luminescence excitation spectra of $\mathrm{LuPO}_{4}-\mathrm{Pr}$ nanoparticles with different size $a$ for $5 d-4 f$ emission of $\operatorname{Pr}^{3+}$ ions $\left(\lambda_{e m}=265 \mathrm{~nm}\right)$. Curves: $1-a=35 \mathrm{~nm} ; 2-$ $a=12 \mathrm{~nm} ; 3-a=8 \mathrm{~nm} ; 3-a<5 \mathrm{~nm} . T=300 \mathrm{~K}$

The structure of the excitation luminescence spectra of praseodymium ions in larger $\mathrm{LuPO}_{4}$-Pr nanoparticles (Fig. 6, curve 1) is identical with that for the single crystal $\mathrm{LuPO}_{4}-\mathrm{Pr}$ [14]. The structure of intracenter absorption bands is changed with the decrease of nanoparticle sizes. This can be observed on band peaked at $5.4 \mathrm{eV}$ that is clearly resolved for nanoparticles with size of $35 \mathrm{~nm}$. However, when the nanoparticle size is reduced the intensity of this excitation band decreases and for nanoparticles smaller than 5 nanometers this band is almost not observed, while the intensive band with maximum at $5.85 \mathrm{eV}$ appears (Fig. 6, curves 1-4). Such change of excitation spectrum could be caused by the different ratio between praseodymium ions located on the surface and in the nanoparticle volume. The praseodymium ions located on the nanoparticle surface have crystalline field surrounding different from that for $\operatorname{Pr}^{3+}$ ions in the nanoparticle volume. Such behavior of luminescence excitation spectra in the range of intracenter absorption was observed for $\mathrm{LuPO}_{4}-\mathrm{Ce}$ nanoparticles also [15].

Decay kinetics of praseodymium emission $\left(\lambda_{\mathrm{em}}=264 \mathrm{~nm}\right)$ excited in the intracenter absorption band of $\operatorname{Pr}^{3+}$ ions $\left(\lambda_{\text {exc }}=180 \mathrm{~nm}\right)$ for $\mathrm{LuPO}_{4}$-Pr nanoparticles with different sizes are shown in Fig. 7. Exponential approximation of decay luminescence kinetics for nanoparticles with mean size $35 \mathrm{~nm}$ gives a time constant about $\tau \sim 7 \mathrm{~ns}$. As can be seen from the figure the luminescence decay kinetics at 
decrease of $\mathrm{LuPO}_{4}$-Pr nanoparticles size become nonexponentional. Approximation by sum of two exponents of curve $4(a<5 \mathrm{~nm})$ reveals decay time constants about 4 ns (26\% of integral intensity) and 13 ns ( $74 \%)$. Such behavior of decay kinetics may indicate that the praseodymium ions located on the nanoparticle surface possess longer time constant.

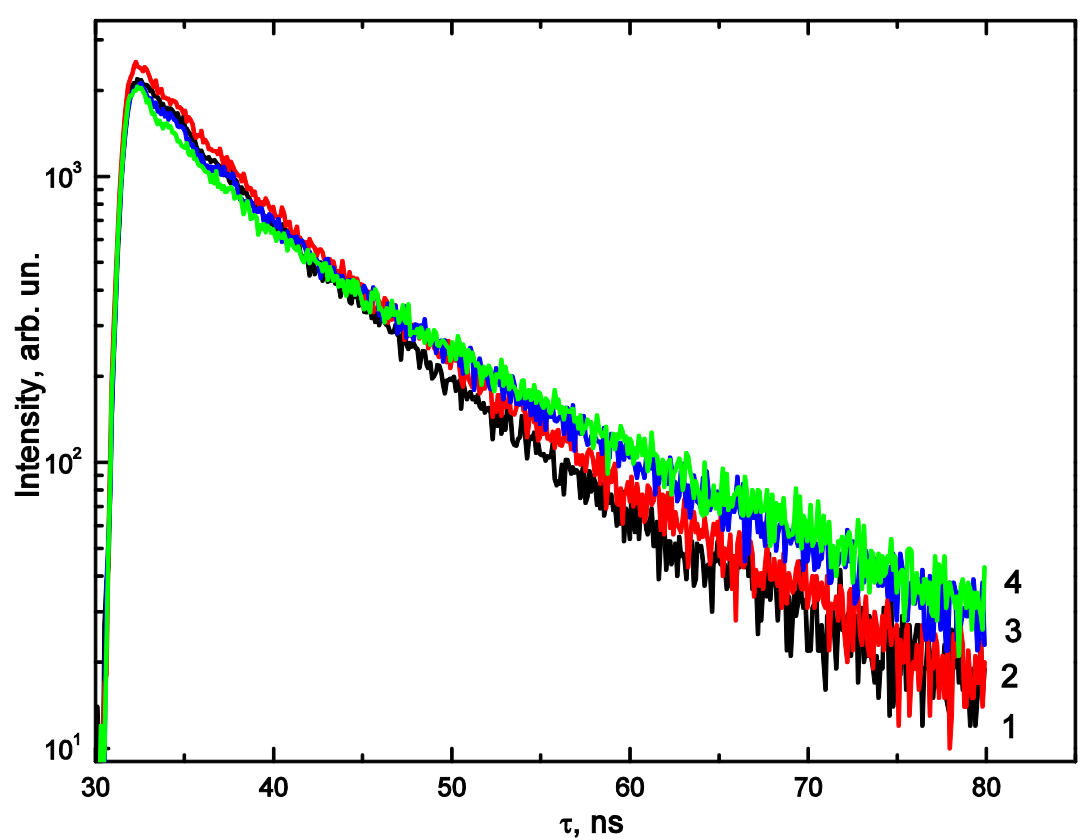

Fig. 7. Decay kinetics of praseodymium luminescence $\left(\lambda_{e m}=264 \mathrm{~nm}\right)$ of $\mathrm{LuPO}_{4}-\mathrm{Pr}$ nanoparticles with different sizes upon excitation in the intracenter absorption band $\left(\lambda_{\text {exc }}=180 \mathrm{~nm}\right)$. Curves: $1-a=35 \mathrm{~nm} ; 2-a=12 \mathrm{~nm} ; 3-a=8 \mathrm{~nm} ; 4-a<5 \mathrm{~nm}$.

Time resolved measurements of luminescence excitation spectra of $\mathrm{Pr}^{3+}$ ions allow to estimate the crystal bandgap. Fig. 8 shows the excitation spectra of fast and slow luminescence components (curves 1 and 2, respectively). The fast component of decay kinetics was measured in the time window 5 ns just after the synchrotron radiation pulse and slow component in $40 \mathrm{~ns}$ time window with the $50 \mathrm{~ns}$ delay after the excitation pulse. One can see the increase of slow time component at $\approx 9 \mathrm{eV}$ is observed, which can be considered as estimation of the bandgap in $\mathrm{LuPO}_{4} \mathrm{Pr}$ nanoparticles. From similar estimation for $\mathrm{LuPO}_{4}-\mathrm{Pr}$ microcrystals the forbidden energy bandgap width value of $8.8 \mathrm{eV}$ was obtained in [14]. 


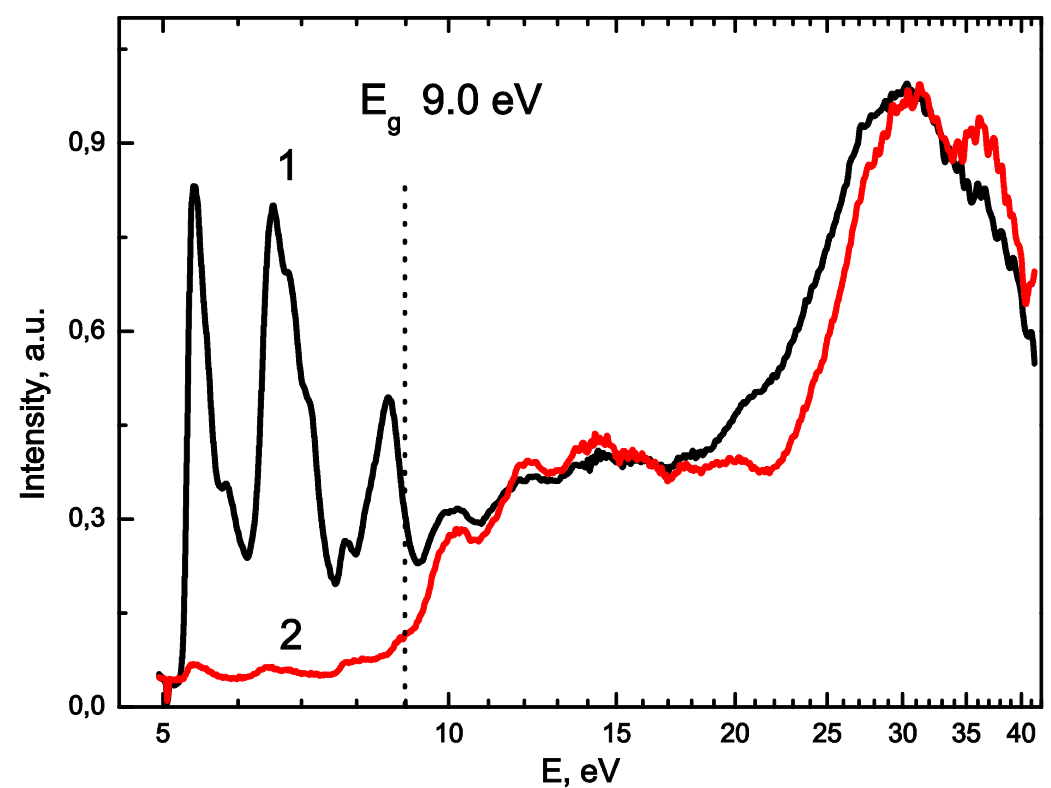

Fig. 8. Luminescence excitation spectra $\left(\lambda_{e m}=264 \mathrm{~nm}\right)$ of $\mathrm{Pr}^{3+}$ ions in $\mathrm{LuPO}_{4}-\mathrm{Pr}$ nanoparticles with mean size of $35 \mathrm{~nm}$ upon registration of fast (1) and slow luminescence decay components (2).

Reducing of the size of $\mathrm{LuPO}_{4}$-Pr nanoparticles causes the luminescence intensity decrease for all excitation energies ( $4 \mathrm{eV}<h v<40 \mathrm{eV}$ ) (Fig. 9). The least sensitive to the nanoparticle size variation is the intensity of luminescence excited in the intracenter absorption band of praseodymium ions (Fig. 9). More significant decrease of the luminescence intensity is observed at the excitation by photons with energies $E_{\mathrm{g}}<h v<2 E_{\mathrm{g}}$, where the recombination mechanism of excitation takes place.

Because the size of intracenter electronic excitation is comparable with praseodymium ion radius $(0.11 \mathrm{~nm})$, the dependence of luminescence intensity upon excitation in the absorption bands of $\operatorname{Pr}^{3+}$ ions on the nanoparticle size will be determined only by their interaction with surface defects. Since the reduce of nanoparticle size increases the ratio between the ions located on the surface and in the nanoparticle volume, the probability of nonradiative relaxation of excitation increases [6] causing the decrease of luminescence intensity. When we move to higher energy of excitation quanta in addition to this the luminescence quenching due to losses on energy transfer from charge carriers to luminescence centers appears. When the $\mathrm{LuPO}_{4}-\mathrm{Pr}$ nanoparticles are excited in the $E_{\mathrm{g}}<h v<2 E_{\mathrm{g}}$ energy range the free electrons and holes are created. They undergo thermalization due to electron-phonon scattering. Thermalization length for electrons can be estimated as tens of nanometers and for holes as several nanometers $[5,16]$. The thermalization process is spatially limited by the size of nanoparticles. If the thermalization length exceeds the size of nanoparticles (that is typical for particles with size $<5 \mathrm{~nm}$ ) the electrons can be trapped on surface defects or can escape the nanoparticle volume. In this case, the intensity of recombination luminescence will decrease significantly. Indeed, the luminescence intensity of $\mathrm{LuPO}_{4}$-Pr nanoparticles excited with energy quanta $h v>E_{\mathrm{g}}$ strongly depends on the size of nanoparticles (Fig. 9, curves 3, 4) and for 
nanoparticles with small size $(a<5 \mathrm{~nm})$ the praseodymium luminescence is practically not excited in this energy range.

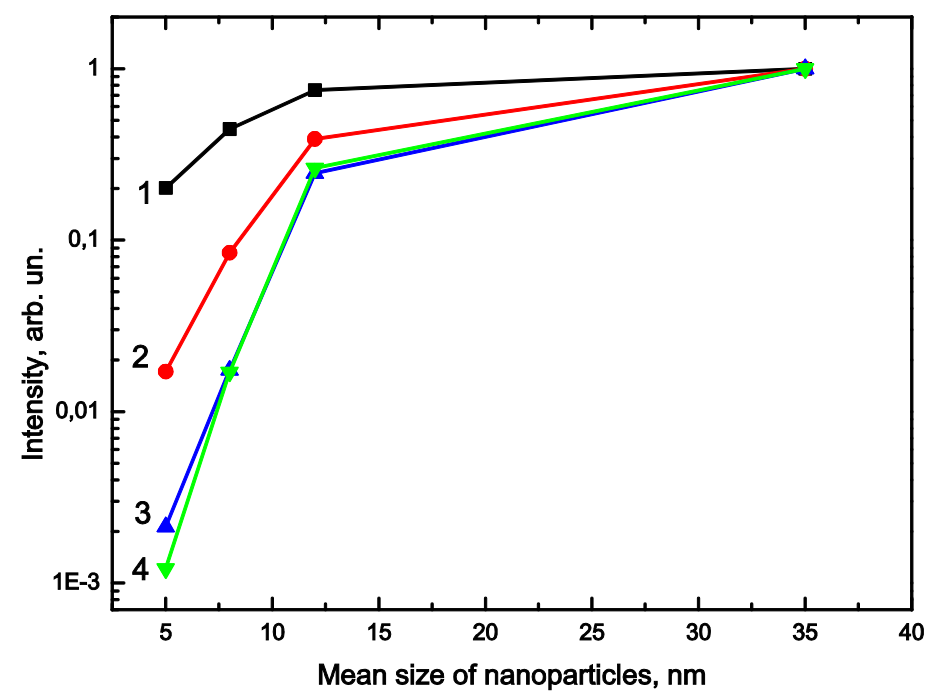

Fig. 9. Dependencies of luminescence intensity in $\mathrm{LuPO}_{4}$-Pr nanoparticles on the size of nanoparticle at various excitation energies: 1 - excitation in the intracenter absorption band of $\operatorname{Pr}^{3+}$ ions $(6.6 \mathrm{eV}) ; 2$ - excitonic band excitation $(8.8 \mathrm{eV}) ; 3-$ excitation in the electron-hole pairs creation range $(11 \mathrm{eV}), 4$ - excitation in the electronic excitation multiplication range $(30 \mathrm{eV})$.

While for small $(\leq 8 \mathrm{~nm}) \quad \mathrm{LuPO}_{4}-\mathrm{Pr}$ nanoparticles the luminescence is practically not excited in the band-to-band transitions range, for nanoparticles $\mathrm{LuPO}_{4}$ Eu with the same size the luminescence excited by photons with energy $h v>E_{\mathrm{g}}$, especially in the range of electronic excitation multiplication, is observed. Thereby, the hole recombination luminescence (when the luminescent center first captures electron then recombines with hole) typical for $\mathrm{LuPO}_{4}$-Eu nanoparticles is less sensitive for nanoparticle size reduce comparing to electron recombination luminescence (where the luminescent center first captures hole then recombines with electron) in $\mathrm{LuPO}_{4}$-Pr nanoparticles. The difference in the mobility of electrons and holes can be the reason of this. In small nanoparticles the impurity centers mainly are localized in the surface layer. During the migration process of hole to the luminescent center (electron recombination luminescence of $\operatorname{Pr}^{3+}$ ions) the electron can be captured by the surface defect or escape the nanoparticle. If the electron is captured first (the hole recombination luminescence of $\mathrm{Eu}^{3+}$ ions) there is a high probability of photoelectron localization on the impurity $\mathrm{Eu}^{3+}$ ions instead of surface defects. Next, the created $\mathrm{Eu}^{2+}$ center due to Coulomb interaction will attract the less mobile hole. Therefore, in the case of space limitation the hole recombination luminescence has more favorable conditions for observation compared to the electronic one.

\section{Conclusions}

$\mathrm{LuPO}_{4}-\mathrm{Eu}$ and $\mathrm{LuPO}_{4}$-Pr nanoparticles of $35 \mathrm{~nm}$ size reveal the luminescence spectra similar to those of microcrystals. The structure of luminescence bands is typical for the tetragonal crystalline symmetry. For nanoparticles with $<5 \mathrm{~nm}$ size the 
luminescence bands are slightly broadened in comparison with the luminescence spectra in samples with larger size $(8-35 \mathrm{~nm})$. This situation is common for nanoparticles and is explained by the fluctuations of the crystal field for impurities in small size nanoparticles.

The intensity of luminescence generally decreases with the decrease of nanoparticles size. It can be explained by the increasing of influence of near surface defects in nanoparticles with smaller sizes. However, the tendencies of luminescence intensity decrease differ for different energy of excitation quanta. Thus, the intensity decrease upon excitation in the charge transfer band $(\sim 6.5 \mathrm{eV})$ for $\mathrm{LuPO}_{4}$-Eu and in the range of $4 f-5 d$ transitions in $\mathrm{Pr}^{3+}$ ions (5.0-7.5 eV) in $\mathrm{LuPO}_{4}$-Pr nanoparticles are not so sharp as in the range of band-to-band transitions. Electronic recombination luminescence in $\mathrm{LuPO}_{4}$-Eu nanoparticles reveals slighter dependence of luminescence intensity on the particle size in comparison with hole recombination luminescence in $\mathrm{LuPO}_{4}$ - $\mathrm{Pr}$ nanoparticles. However, the luminescence intensity of $\mathrm{LuPO}_{4}$-Eu and $\mathrm{LuPO}_{4}-\mathrm{Pr}$ nanoparticles significantly decreases with the reducing of nanoparticle size in the recombination excitation range in comparison with the excitation in the intracenter absorption range. This is due to the fact that electrons created by the recombination excitation during thermalization process can reach the surface and escape nanoparticle volume or nonradiatively relax due to interaction with surface defects. The probability of such processes increases with the reducing of the ratio between the size of nanoparticle and the electron thermalization length.

\section{Acknowledgement}

The work is partially supported by Ukraine Ministry of Science and Education (grant No. 0115U003251).

[1] N.Y. Morgan, G. Kramer-Marek, P.D. Smith, K. Camphausen, J. Capala, Nanoscintillator conjugates as photodynamic therapy-based radiosensitizers: calculation of required physical parameters., Radiat. Res. 171 (2009) 236-44. doi:10.1667/RR1470.1.

[2] E.A. McKigney, R.E. Del Sesto, L.G. Jacobsohn, P.A. Santi, R.E. Muenchausen, K.C. Ott, T. Mark McCleskey, B.L. Bennett, J.F. Smith, D. Wayne Cooke, Nanocomposite scintillators for radiation detection and nuclear spectroscopy, Nucl. Instruments Methods Phys. Res. Sect. A Accel.

Spectrometers, Detect. Assoc. Equip. 579 (2007) 15-18.

doi:10.1016/j.nima.2007.04.004.

[3] G. Stryganyuk, D.M. Trots, A. Voloshinovskii, T. Shalapska, V. Zakordonskiy, V. Vistovskyy, M. Pidzyrailo, G. Zimmerer, Luminescence of $\mathrm{Ce}^{3+}$ doped $\mathrm{LaPO}_{4}$ nanophosphors upon Ce ${ }^{3+} 4 \mathrm{f}-5 \mathrm{~d}$ and band-to-band excitation, J. Lumin. 128 (2008) 355-360.

[4] A.V.G. Andrey N. Vasil'ev, Multiscale Approach to Estimation of Scintillation Characteristics, IEEE Trans. Nucl. Sci. 61 (2014) 235 - 245. doi:10.1109/TNS.2013.2282117.

[5] G. Bizarri, W.W. Moses, J. Singh, A.N. Vasil'Ev, R.T. Williams, An analytical model of nonproportional scintillator light yield in terms of recombination rates, J. Appl. Phys. 105 (2009) 044507. doi:10.1063/1.3081651. 
[6] L.G. Jacobsohn, K.B. Sprinkle, S.A. Roberts, C.J. Kucera, T.L. James, E.G. Yukihara, T.A. DeVol, J. Ballato, Fluoride Nanoscintillators, J. Nanomater. 2011 (2011) 1-6. doi:10.1155/2011/523638.

[7] V.N. Makhov, C. Lushchik, A. Lushchik, M. Kirm, Z.F. Wang, W.P. Zhang, M. Yin, J.T. Zhao, Multiplication of electronic excitations in nanophosphors $\mathrm{Lu}_{2} \mathrm{O}_{3}: \mathrm{Eu}^{3+}$ and $\mathrm{Lu}_{2} \mathrm{O}_{3}: \mathrm{Tb}^{3+}$, J. Lumin. 129 (2009) 1711-1714. doi:10.1016/j.jlumin.2008.12.028.

[8] V. Pankratov, A.I. Popov, L. Shirmane, A. Kotlov, C. Feldmann, $\mathrm{LaPO}_{4}$ :Ce,Tb and $\mathrm{YVO}_{4}$ :Eu nanophosphors: Luminescence studies in the vacuum ultraviolet spectral range, J. Appl. Phys. 110 (2011) 053522.

doi:http://dx.doi.org/10.1063/1.3634112.

[9] V. V Vistovskyy, A. V Zhyshkovych, N.E. Mitina, A.S. Zaichenko, A. V Gektin, A.N. Vasil'ev, A.S. Voloshinovskii, Relaxation of electronic excitations in $\mathrm{CaF}_{2}$ nanoparticles, J. Appl. Phys. 112 (2012) 024325. doi:http://dx.doi.org/10.1063/1.4739488.

[10] T.S. Malyy, V. V Vistovskyy, Z.A. Khapko, A.S. Pushak, N.E. Mitina, A.S. Zaichenko, A. V Gektin, A.S. Voloshinovskii, Recombination luminescence of $\mathrm{LaPO}_{4}$-Eu and $\mathrm{LaPO}_{4}$-Pr nanoparticles, J. Appl. Phys. 113 (2013) 224305. doi:http://dx.doi.org/10.1063/1.4808797.

[11] V. Vistovskyy, N. Mitina, A. Shapoval, T. Malyy, A. Gektin, T. Konstantinova, A. Voloshinovskii, A. Zaichenko, Luminescence properties of $\mathrm{LaPO}_{4}-\mathrm{Eu}$ nanoparticles synthesized in the presence of surface active oligoperoxide as template, in: Opt. Mater. (Amst)., 2012: pp. 2066-2070.

[12] G. Zimmerer, SUPERLUMI: A unique setup for luminescence spectroscopy with synchrotron radiation, Radiat. Meas. 42 (2007) 859-864. doi:10.1016/j.radmeas.2007.02.050.

[13] C. Dujardin, D. Amans, A. Belsky, F. Chaput, G. Ledoux, A. Pillonnet, Luminescence and Scintillation Properties at the Nanoscale, IEEE Trans. Nucl. Sci. 57 (2010) 1348-1354. doi:10.1109/TNS.2009.2035697.

[14] V. V. Mikhailin, D.A. Spassky, V.N. Kolobanov, A.A. Meotishvili, D.G. Permenov, B.I. Zadneprovski, Luminescence study of the $\mathrm{LuBO}_{3}$ and $\mathrm{LuPO}_{4}$ doped with $\mathrm{RE}^{3+}$, Radiat. Meas. 45 (2010) 307-310. doi:10.1016/j.radmeas.2009.12.019.

[15] V. Vistovskyy, T. Malyy, A. Pushak, A. Vas'Kiv, A. Shapoval, N. Mitina, A. Gektin, A. Zaichenko, A. Voloshinovskii, Luminescence and scintillation properties of $\mathrm{LuPO}_{4}$-Ce nanoparticles, J. Lumin. 145 (2014) 232-236.

[16] Z. Wang, Y. Xie, L.W. Campbell, F. Gao, S. Kerisit, Monte Carlo simulations of electron thermalization in alkali iodide and alkaline-earth fluoride scintillators, J. Appl. Phys. 112 (2012) 014906. doi:10.1063/1.4736088. 